\title{
Emergency Tracheostomy in Two Airway Trauma Patients Suspected of COVID-19: A Case Report
}

\author{
Valiollah Hassani ${ }^{1}$, Saied Amniati (iD) ${ }^{1,}{ }^{*}$, Aslan Ahmadi ${ }^{2}$, Masood Mohseni ${ }^{(i)}{ }^{1}$, Saloome Sehat-Kashani ${ }^{1}$, \\ Nasim Nikoubakht ${ }^{1}$, Pooya Derakhshan (iD) ${ }^{1}$, Reza Farahmand Rad $^{1}$ and Azadeh Habibi ${ }^{1}$ \\ ${ }^{1}$ Pain Research Center, Iran University of Medical Sciences, Tehran, Iran \\ ${ }^{2}$ ENt and Head and Neck Research Centerand Department, The Five Sences Institue, Hazrat Rasoul Hospital, Iran University of Medical Sciences, Tehran, Iran \\ "Corresponding author: Pain Research Center, Iran University of Medical Sciences, Tehran, Iran. Email: saiedamniati@gmail.com
}

Received 2020 May 06; Revised 2020 June 17; Accepted 2020 June 23.

\begin{abstract}
Coronavirus disease 2019, known as COVID-19, was first identified in Wuhan, China, in December 2019 and became a pandemic on Mar 11, according to the World Health Organization report. In the epidemic of COVID-19, many patients admitted to hospitals for other reasons may be silent carriers of COVID-19 and have the risk of infecting medical personnel. Thus, meticulous personal protection measures should be considered in suspicious patients, especially when close contact with the patient's airway is anticipated. We introduce two airway trauma patients suspected of COVID-19 who required emergency tracheostomy. Patient one was a 29-year-old man who suffered facial trauma following a car accident. A chest CT scan showed peripheral ground-glass opacities suggestive for COVID-19. The second patient was a young elevator mechanic who experienced maxillofacial trauma after an elevator crash. The methods of anesthesia and airway protection and safety precautions are described.
\end{abstract}

Keywords: Airway Management, Coronavirus, COVID-19, Anesthesia

\section{Introduction}

Coronavirus disease 2019 (COVID-19) was first identified in December 2019 in Wuhan, Hubei, China. Since then, it has spread globally, resulting in the ongoing 2019 2020 coronavirus pandemic. It has affected more than 2.5 million people worldwide up to the time of writing this manuscript (1). Iran was one of the involved countries with critical outbreaks in some provinces. The virus is primarily spread between people during close contact often via small droplets produced by coughing, sneezing, or talking. People may also become infected by touching a contaminated surface and then touching their face. Physicians and other health care workers who perform examinations and participate in procedures within the head and neck region and airways are particularly at high risk of exposure and infection from aerosol and droplet contamination. Many patients who come to the hospital during a pandemic may be asymptomatic carriers of the coronavirus. In this manuscript, we introduce two airway trauma patients suspected of COVID-19 who required emergency tracheostomy. The report was prepared after the patients' consent for publication.

\section{Case Presentation}

\subsection{Case 1}

A 29-year-old man suffered facial trauma following a car accident. The patient was transferred by road emergency to the emergency center of Rasoul Akram Hospital, Tehran. After the primary assessment and preparation, the patient was transferred to the operating room. In the examination of the patient, upper and lower jaw trauma with deformity, extensive detachment of the skin, and mucous membranes with active bleeding were obvious. The patient was conscious but agitated with a stable hemodynamic profile. $\mathrm{SPO}_{2}$ was $98 \%$, with oxygen delivered via a mask. Respiratory distress was mild, but ongoing airway obstruction due to edema and bleeding was a great concern. The patient could not lie down because of bleeding and a feeling of suffocation.

The anesthetist and ENT surgeon decided to perform awake tracheostomy in the semi-sitting position. However, the patient was not cooperative, and the anesthetist decided to sedate the patient while maintaining spontaneous breathing. After standard monitoring and checking for IV access, he was sedated with $100 \mu \mathrm{g}$ of fentanyl and $30 \mathrm{mg}$ of propofol. After local anesthesia with lidocaine 1\%, 
tracheostomy was performed immediately and uneventful. Further titrated doses of propofol up to $40 \mathrm{mg}$ were required to maintain the patient sedated. After end-tidal $\mathrm{CO}_{2}$ $\left(\mathrm{ETCO}_{2}\right)$ monitoring and ensuring the secure airway, the induction of anesthesia and muscle relaxation with propofol and cisatracurium were applied. The soft tissue bleeding was repaired and the team decided to perform the secondary repair after stabilization and further radiologic assessment. Finally, muscle relaxation was reversed and the patient transferred to the ward in good general condition.

In CT scans, multiple fractures in upper and lower jaws, as well as ground-glass opacities in the periphery of the lungs, were noticed (Figure 1). The patient was discharged from neurosurgery and general surgery services and a consultation with the infectious diseases department was requested. Hydroxy chloroquine and oseltamivir started for him according to the national protocol at the time of diagnosis. APCR test was requested for COVID-19, which demonstrated infection with the coronavirus. The course of treatment was uneventful and the patient was discharged two weeks later after the repair of jaw fractures and tracheostomy closure.

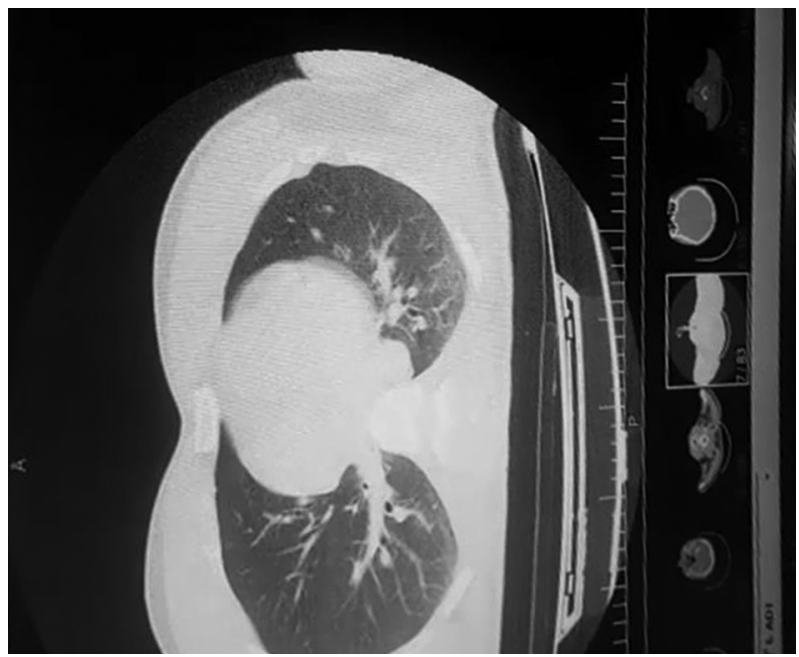

Figure 1. Chest CT scan with ground glass opacities suggestive of the coronavirus

\subsection{Case 2}

The second patient was a 47-year-old elevator mechanic who experienced maxillofacial trauma after an elevator crash. The patient was conscious, experiencing moderate degrees of respiratory distress with the following vital signs: $\mathrm{BP}=138 / 76, \mathrm{PR}=130$, and $\mathrm{SPO}_{2}=92 \%$ with oxygenation via nasal cannula. Because of a poor laryngoscopic view and upcoming airway collapse due to massive orofa- cial bleeding, tracheostomy with local lidocaine $1 \%$ was attempted. During the procedure, the patient experienced one episode of bradycardia while opening the window for the tracheostomy tube. The event was treated with immediate atropine injection. After confirmation of the secure airway, the patient was pre-medicated with fentanyl and anesthetized with sodium thiopental and cisatracurium. To compensate for blood loss and volume contraction, two units of packed RBC and three liters of isotonic crystalloids were infused. Serial blood gas analyses were within an acceptable range. Considering the outbreak of the coronavirus and because of $\mathrm{SPO}_{2}$ of less than anticipated, the patient was suspected of having COVID-19. Thus, personal protective measures, including N95 respirators, face shields, and double gloves were administered by the team in close contact with the patient. However, postoperative laboratory and radiologic assessments, including PCR for COVID-19 and chest CT scan ruled out the infection. The auscultation of the lung field in the early postoperative period was coarse but gradually improved with airway toilet, which suggested micro-aspiration of blood and secretions in the preoperative period. The patient was discharged one week later after jaw fracture fixation and secondary repair of soft tissues.

\section{Discussion}

COVID-19 was diagnosed in December 2019 in Wuhan, China, and became a pandemic at the beginning of 2020 . Most patients have asymptomatic presentations or mild disease (2). During the epidemic, many asymptomatic patients who come to hospitals for other reasons may be asymptomatic carriers of the disease. Healthcare workers in the forehead of the disease control have a higher risk of being infected than the general population. Many medical procedures such as endotracheal intubation, mask ventilation, and tracheostomy carry high risks of transmission; thus, it is necessary to implement preventive strategies (3).

The airway can be threatened by a variety of reasons, one of which is trauma. In dealing with difficult airways, assistant devices such as fiberoptic bronchoscope and video-laryngoscope may be used in either awake or anesthetized patients (4). In maxillofacial trauma, mask ventilation and endotracheal intubation could be difficult. The risk of airway obstruction in bilateral mandibular fractures is significant. Cervical spine instability and airway bleeding are common conditions that exacerbate the condition. The latter prohibits the applicability of fiberoptic bronchoscopy or even direct laryngoscopy (5). A relatively safe alternative method is tracheostomy under local anesthesia. 
Endotracheal intubation and tracheostomy are known as aerosol-producing procedures. Therefore, they may facilitate the transmission of COVID-19. The goal is to minimize the time of exposure to aerosolized secretions intraoperatively. This may be achieved by some preventive strategies. If the patient is intubated and tracheostomy is to be performed, the patient must be sufficiently relaxed and receive an appropriate depth of anesthesia to prevent coughing and shedding the virus (6). Immediately before entering the endotracheal tube, positive pressure should not be performed and mechanical ventilation should stop $(7,8)$. During the procedure, the use of suctions should be reduced and if available, a closed system with a filter should be used $(8,9)$. Medications such as remifentanil infusion may be used to reduce coughing and thus decrease the risk of contamination (10). Cuffed non-fenestrated tracheostomy tubes are most preferred. Finally, the indication of tracheostomy should be carefully reviewed and, if possible, delayed for 14 days to reduce the risk of contamination. Indeed, an early tracheostomy cannot reduce the length of ICU stay (11).

In emergency tracheostomy, especially in an awake patient, such as our case, most of the above-mentioned preventive strategies are not applicable. Measures to reduce the possibility of contamination include appropriate hand hygiene before and after the procedure and wearing gown, double-gloves, face shields, and a fit-tested N95 respirator or an equivalent effective mask (6). Finally, we should remember that personal protection is the priority. When performing aerosol-producing procedures during the pandemic of the coronavirus, every patient should be considered suspicious.

\section{Footnotes}

Authors' Contribution: Study conception and design: Valiollah Hassani and Aslan Ahmadi. Acquisition of data: Saloome Sehat-Kashani, and Nasim Nikoubakht. Analysis and interpretation of data: Pooya Derakhshan. Drafting of the manuscript: Reza Farahmand Rad and Azadeh Habibi. Critical revision: Saied Amniati and Masood Mohseni.

Conflict of Interests: There is no conflict of interest.
Funding/Support: This study received no grants or support.

Informed Consent: The report was prepared after patients' consent for publication.

\section{References}

1. Pergolizzi Jr JV, Magnusson P, LeQuang J JA, Breve F, Paladini $A$, Rekatsina $M$, et al. The Current clinically relevant findings on COVID-19 Pandemic. Anesth Pain Med. 2020;10(2). 103819. doi: 10.5812/aapm.103819.

2. Hui DS, I. Azhar E, Madani TA, Ntoumi F, Kock R, Dar O, et al. The continuing 2019-nCoV epidemic threat of novel coronaviruses to global health - The latest 2019 novel coronavirus outbreak in Wuhan, China. Int J Infect Dis. 2020;91:264-6. doi:10.1016/j.ijid.2020.01.009. [PubMed: 31953166]. [PubMed Central: PMC7128332].

3. Centers for Disease Control and Prevention. Interim infection prevention and control recommendations for patients with confirmed (COVID-19) or persons under investigation for COVID-19 in healthcare setting. 2020, [cited 2020 May 18]. Available from: http://www.cdc.govcoronavirus/ 2019-ncov.

4. Hassani V, Faiz S, Rahimzadeh P, Sharifian M. Fiberoptic intubation. RJMS. 2012;19(97):17-27.

5. Barak M, Bahouth H, Leiser Y, Abu El-Naaj I. Airway management of the patient with maxillofacial trauma: Review of the literature and suggested clinical approach. Biomed Res Int. 2015;2015:724032. doi: 10.1155/2015/724032. [PubMed: 26161411]. [PubMed Central: PMC4486512].

6. Kwan A, Fok WG, Law KI, Lam SH. Tracheostomy in a patient with severe acute respiratory syndrome. Br J Anaesth. 2004;92(2):2802. doi: 10.1093/bja/aeh035. [PubMed: 14722185]. [PubMed Central: PMC7094700].

7. Zhao S, Ling $\mathrm{K}$, Yan $\mathrm{H}$, Zhong L, Peng $\mathrm{X}$, Yao S, et al. Anesthetic management of patients with COVID 19 infections during emergency procedures. J Cardiothorac Vasc Anesth. 2020;34(5):1125-31. doi: 10.1053/j.jvca.2020.02.039. [PubMed: 32178954]. [PubMed Central: PMC7102598].

8. Tay JK, Khoo ML, Loh WS. Surgical considerations for tracheostomy during the COVID-19 Pandemic: Lessons learned from the severe acute respiratory syndrome outbreak. JAMA Otolaryngol Head Neck Surg. 2020. doi:10.1001/jamaoto.2020.0764. [PubMed:32232426].

9. Seyfi S, Hassani V, Faiz SHR, Latifi S, Hajiesmaeili MR, Sedaghat A, et al. Surgical tracheostomy versus percutaneous tracheostomy in the intensive care unit. Razi J Med Sci. 2013;19(104):29-33.

10. Ghodraty MR, Hasani V, Bagheri-Aghdam A, Zamani MM, Pournajafian A, Rokhtabnak F, et al. Remifentanil infusion during emergence moderates hemodynamic and cough responses to the tracheal tube: A randomized controlled trial. J Clin Anesth. 2016;33:514-20. doi: 10.1016/j.jclinane.2015.09.001. [PubMed: 26603110].

11. Givi B, Schiff BA, Chinn SB, Clayburgh D, Iyer NG, Jalisi S, et al. Safety recommendations for evaluation and surgery of the head and neck during the COVID-19 pandemic. JAMA Otolaryngol Head Neck Surg. 2020. doi: 10.1001/jamaoto.2020.0780. [PubMed: 32232423]. 